PROCEEDINGS OF THE

AMERICAN MATHEMATICAL SOCIETY

Volume 125, Number 7, July 1997, Pages 2051-2055

S $0002-9939(97) 03818-5$

\title{
FACTORS FROM TREES
}

\author{
JACQUI RAMAGGE AND GUYAN ROBERTSON
}

(Communicated by Palle E. T. Jorgensen)

\begin{abstract}
We construct factors of type $\mathrm{III}_{1 / n}$ for $n \in \mathbb{N}, n \geq 2$, from group actions on homogeneous trees and their boundaries. Our result is a discrete analogue of a result of R.J Spatzier, where the hyperfinite factor of type III $_{1}$ is constructed from a group action on the boundary of the universal cover of a manifold.
\end{abstract}

\section{INTRODUCTION}

Let $\Gamma$ be a group acting simply transitively on the vertices of a homogeneous tree $\mathcal{T}$ of degree $n+1<\infty$. Then, by [FTN, Ch. I, Theorem 6.3],

$$
\Gamma \cong \mathbb{Z}_{2} * \cdots * \mathbb{Z}_{2} * \mathbb{Z} * \cdots * \mathbb{Z}
$$

where there are $s$ factors of $\mathbb{Z}_{2}, t$ factors of $\mathbb{Z}$, and $s+2 t=n+1$. Thus $\Gamma$ has a presentation

$$
\Gamma=\left\langle a_{1}, \ldots, a_{s+t}: a_{i}^{2}=1 \text { for } i \in\{1, \ldots, s\}\right\rangle,
$$

we can identify the Cayley graph of $\Gamma$ constructed via right multiplication with $\mathcal{T}$ and the action of $\Gamma$ on $\mathcal{T}$ is equivalent to the natural action of $\Gamma$ on its Cayley graph via left multiplication.

We can associate a natural boundary to $\mathcal{T}$, namely the set $\Omega$ of semi-infinite reduced words in the generators of $\Gamma$. The action of $\Gamma$ on $\mathcal{T}$ induces an action of $\Gamma$ on $\Omega$.

For each $x \in \Gamma$, let

$$
\Omega^{x}=\{\omega \in \Omega: \omega=x \cdots\}
$$

be the set of semi-infinite reduced words beginning with $x$. The set $\left\{\Omega^{x}\right\}_{x \in \Gamma}$ is a set of basic open sets for a compact Hausdorff topology on $\Omega$. Denote by $|x|$ the length of a reduced expression for $x$. Let $V^{m}=\{x \in \Gamma:|x|=m\}$ and define $N_{m}=\left|V^{m}\right|$. Then $\Omega$ is the disjoint union of the $N_{m}$ sets $\Omega^{x}$ for $x \in V^{m}$.

We can also endow $\Omega$ with the structure of a measure space. $\Omega$ has a unique distinguished Borel probability measure $\nu$ such that

$$
\nu\left(\Omega^{x}\right)=\frac{1}{n+1}\left(\frac{1}{n}\right)^{|x|-1}
$$

for every nontrivial $x \in \Gamma$. The sets $\Omega^{x}, x \in \Gamma$, generate the Borel $\sigma$-algebra.

Received by the editors January 26, 1996.

1991 Mathematics Subject Classification. Primary 46L10.

(C)1997 American Mathematical Society 
This measure $\nu$ on $\Omega$ is quasi-invariant under the action of $\Gamma$, so that $\Gamma$ acts on the measure space $(\Omega, \nu)$ and enables us to extend the action of $\Gamma$ to an action on $L^{\infty}(\Omega, \nu)$ via

$$
g \cdot f(\omega)=f\left(g^{-1} \cdot \omega\right)
$$

for all $g \in \Gamma, f \in L^{\infty}(\Omega, \nu)$, and $\omega \in \Omega$. We may therefore consider the von Neumann algebra $L^{\infty}(\Omega, \nu) \rtimes \Gamma$ which we shall write as $L^{\infty}(\Omega) \rtimes \Gamma$ for brevity.

\section{THE FACTORS}

We note that the action of $\Gamma$ on $\Omega$ is free since if $g \omega=\omega$ for some $g \in \Gamma$ and $\omega \in \Omega$ then we must have either $\omega=g g g \cdots$ or $\omega=g^{-1} g^{-1} g^{-1} \cdots$ and

$$
\nu\left\{g g g \cdots, g^{-1} g^{-1} g^{-1} \cdots\right\}=0 .
$$

The action of $\Gamma$ on $\Omega$ is also ergodic by the proof of [PS, Proposition 3.9], so that $L^{\infty}(\Omega) \rtimes \Gamma$ is a factor. Establishing the type of the factor is not quite as straightforward. We begin by recalling some classical definitions.

Definition 2.1. Given a group $\Gamma$ acting on a measure space $\Omega$, we define the full group, $[\Gamma]$, of $\Gamma$ by

$$
[\Gamma]=\{T \in \operatorname{Aut}(\Omega): T \omega \in \Gamma \omega \text { for almost every } \omega \in \Omega\} .
$$

The set $[\Gamma]_{0}$ of measure preserving maps in $[\Gamma]$ is then given by

$$
[\Gamma]_{0}=\{T \in[\Gamma]: T \circ \nu=\nu\} .
$$

Definition 2.2. Let $G$ be a countable group of automorphisms of the measure space $(\Omega, \nu)$. Following W. Krieger, define the ratio set $r(G)$ to be the subset of $[0, \infty)$ such that if $\lambda \geq 0$ then $\lambda \in r(G)$ if and only if for every $\epsilon>0$ and Borel set $\mathcal{E}$ with $\nu(\mathcal{E})>0$, there exist a $g \in G$ and a Borel set $\mathcal{F}$ such that $\nu(\mathcal{F})>0$, $\mathcal{F} \cup g \mathcal{F} \subseteq \mathcal{E}$ and

$$
\left|\frac{d \nu \circ g}{d \nu}(\omega)-\lambda\right|<\epsilon
$$

for all $\omega \in \mathcal{F}$.

Remark 2.3. The ratio set $r(G)$ depends only on the quasi-equivalence class of the measure $\nu$; see [HO, $\S$ I-3, Lemma 14]. It also depends only on the full group in the sense that

$$
[H]=[G] \Rightarrow r(H)=r(G)
$$

The following result will be applied in the special case where $G=\Gamma$. However, since the simple transitivity of the action doesn't play a role in the proof, we can state it in greater generality.

Proposition 2.4. Let $G$ be a countable subgroup of $\operatorname{Aut}(\mathcal{T}) \leq \operatorname{Aut}(\Omega)$. Suppose there exist an element $g \in G$ such that $d(g e, e)=1$ and a subgroup $K$ of $[G]_{0}$ whose action on $\Omega$ is ergodic. Then

$$
r(G)=\left\{n^{k}: k \in \mathbb{Z}\right\} \cup\{0\} .
$$

Proof. By Remark 2.3, it is sufficient to prove the statement for some group $H$ such that $[H]=[G]$. In particular, since $[G]=[\langle G, K\rangle]$ for any subgroup $K$ of $[G]_{0}$, we may assume without loss of generality that $K \leq G$.

By [FTN, Chapter II, part 1)], for each $g \in G$ and $\omega \in \Omega$ we have

$$
\frac{d \nu \circ g}{d \nu}(\omega) \in\left\{n^{k}: k \in \mathbb{Z}\right\} \cup\{0\} .
$$


Since $G$ acts ergodically on $\Omega, r(G) \backslash\{0\}$ is a group. It is therefore enough to show that $n \in r(G)$. Write $x=g e$ and note that $\nu_{x}=\nu \circ g^{-1}$. By [FTN, Chapter II, part $1)$ ] we have

$$
\frac{d \nu_{x}}{d \nu}(\omega)=n, \text { for all } \omega \in \Omega_{e}^{x} .
$$

Let $\mathcal{E} \subseteq \Omega$ be a Borel set with $\nu(\mathcal{E})>0$. By the ergodicity of $K$, there exist $k_{1}, k_{2} \in K$ such that the set

$$
\mathcal{F}=\left\{\omega \in \mathcal{E}: k_{1} \omega \in \Omega_{e}^{x} \text { and } k_{2} g^{-1} k_{1} \omega \in \mathcal{E}\right\}
$$

has positive measure.

Finally, let $t=k_{2} g^{-1} k_{1} \in G$. By construction, $\mathcal{F} \cup t \mathcal{F} \subseteq \mathcal{E}$. Moreover, since $K$ is measure-preserving,

$$
\frac{d \nu \circ t}{d \nu}(\omega)=\frac{d \nu \circ g^{-1}}{d \nu}\left(k_{1} \omega\right)=\frac{d \nu_{x}}{d \nu}\left(k_{1} \omega\right)=n \text { for all } \omega \in \mathcal{F}
$$

by (1), since $k_{1} \in \Omega_{e}^{x}$. This proves $n \in r(G)$, as required.

Corollary 2.5. If, in addition to the hypotheses for Proposition 2.4, the action of $G$ is free, then $L^{\infty}(\Omega) \rtimes G$ is a factor of type $\mathrm{III}_{1 / n}$.

Proof. Having determined the ratio set, this is immediate from [C1, Corollaire 3.3.4].

Thus, if we can find a countable subgroup $K \leq[\Gamma]_{0}$ whose action on $\Omega$ is ergodic we will have shown that $L^{\infty}(\Omega) \rtimes \Gamma$ is a factor of type $\operatorname{III}_{1 / n}$. To this end, we prove the following sufficiency condition for ergodicity.

Lemma 2.6. Let $K$ be group which acts on $\Omega$. If $K$ acts transitively on the collection of sets $\left\{\Omega^{x}: x \in \Gamma,|x|=m\right\}$ for each natural number $m$, then $K$ acts ergodically on $\Omega$.

Proof. Suppose that $X_{0} \subseteq \Omega$ is a Borel set which is invariant under $K$ and such that $\nu\left(X_{0}\right)>0$. We show that this necessarily implies $\nu\left(\Omega \backslash X_{0}\right)=0$, thus establishing the ergodicity of the action.

Define a new measure $\mu$ on $\Omega$ by $\mu(X)=\nu\left(X \cap X_{0}\right)$ for each Borel set $X \subseteq \Omega$. Now, for each $g \in K$,

$$
\begin{aligned}
\mu(g X) & =\nu\left(g X \cap X_{0}\right)=\nu\left(X \cap g^{-1} X_{0}\right) \\
& =\nu\left(X \cap X_{0}\right) \\
& =\mu(X),
\end{aligned}
$$

and therefore $\mu$ is $K$-invariant. Since $K$ acts transitively on the basic open sets $\Omega^{x}$ associated to words $x$ of length $m$ this implies that

$$
\mu\left(\Omega^{x}\right)=\mu\left(\Omega^{y}\right)
$$

whenever $|x|=|y|$. Since $\Omega$ is the union of $N_{m}$ disjoint sets $\Omega^{x}, x \in V^{m}$, each of which has equal measure with respect to $\mu$, we deduce that

$$
\mu\left(\Omega^{x}\right)=\frac{c}{N_{m}}
$$

for each $x \in V^{m}$, where $c=\mu\left(X_{0}\right)=\nu\left(X_{0}\right)>0$. Thus $\mu\left(\Omega^{x}\right)=c \nu\left(\Omega^{x}\right)$ for every $x \in \Gamma$. 
Since the sets $\Omega^{x}, x \in \Gamma$ generate the Borel $\sigma$-algebra, we deduce that $\mu(X)=$ $c \nu(X)$ for each Borel set $X$. Therefore

$$
\begin{aligned}
\nu\left(\Omega \backslash X_{0}\right) & =c^{-1} \mu\left(\Omega \backslash X_{0}\right) \\
& =c^{-1} \nu\left(\left(\Omega \backslash X_{0}\right) \cap X_{0}\right)=0,
\end{aligned}
$$

thus proving ergodicity.

In the last of our technical results, we give a constructive proof of the existence of a countable ergodic subgroup of $[\Gamma]_{0}$.

Lemma 2.7. There is a countable ergodic group $K \leq \operatorname{Aut}(\Omega)$ such that $K \leq[\Gamma]_{0}$.

Proof. Let $x, y \in V^{m}$. We construct a measure preserving automorphism $k_{x, y}$ of $\Omega$ such that

(1) $k_{x, y}$ is almost everywhere a bijection from $\Omega^{x}$ onto $\Omega^{y}$,

(2) $k_{x, y}$ is the identity on $\Omega \backslash\left(\Omega^{x} \cup \Omega^{y}\right)$.

It then follows from Lemma 2.6 that the group

$$
K=\left\langle k_{x, y}:\{x, y\} \subseteq V^{m}, m \in \mathbb{N}\right\rangle
$$

acts ergodically on $\Omega$ and the construction will show explicitly that $K \leq[\Gamma]_{0}$.

Fix $x, y \in V^{m}$ and suppose that we have reduced expressions $x=x_{1} \ldots x_{m}$, and $y=y_{1} \ldots y_{m}$.

Define $k_{x, y}$ to be left multiplication by $y x^{-1}$ on each of the sets $\Omega^{x z}$ where $|z|=1$ and $z \notin\left\{x_{m}^{-1}, y_{m}^{-1}\right\}$. Then $k_{x, y}$ is a measure preserving bijection from each such set onto $\Omega^{y z}$. If $y_{m}=x_{m}$ then $k_{x, y}$ is now well defined everywhere on $\Omega^{x}$.

Suppose now that $y_{m} \neq x_{m}$. Then $k_{x, y}$ is defined on the set $\Omega^{x} \backslash \Omega^{x y_{m}^{-1}}$, which it maps bijectively onto $\Omega^{y} \backslash \Omega^{y x_{m}^{-1}}$. Now define $k_{x, y}$ to be left multiplication by $y x_{m}^{-1} y_{m} x^{-1}$ on each of the sets $\Omega^{x y_{m}^{-1} z}$ where $|z|=1$ and $z \notin\left\{x_{m}, y_{m}\right\}$. Then $k_{x, y}$ is a measure preserving bijection of each such $\Omega^{x y_{m}^{-1} z}$ onto $\Omega^{y x_{m}^{-1} z}$.

Thus we have extended the domain of $k_{x, y}$ so that it is now defined on the set $\Omega^{x} \backslash \Omega^{x y_{m}^{-1} x_{m}}$, which it maps bijectively onto $\Omega^{y} \backslash \Omega^{y x_{m}^{-1} y_{m}}$.

Next define $k_{x, y}$ to be left muliplication by $y x_{m}^{-1} y_{m} x_{m}^{-1} y_{m} x^{-1}$ on the sets $\Omega^{x y_{m}^{-1} x_{m} z}$ where $|z|=1$ and $z \notin\left\{x_{m}^{-1}, y_{m}^{-1}\right\}$.

Continue in this way. At the $j$ th step $k_{x, y}$ is a measure preserving bijection from $\Omega^{x} \backslash X_{j}$ onto $\Omega^{y} \backslash Y_{j}$ where $\nu\left(X_{j}\right) \rightarrow 0$ as $j \rightarrow \infty$ so that eventually $k_{x, y}$ is defined almost everywhere on $\Omega$. Finally, define

$$
k_{x, y}\left(x y_{m}^{-1} x_{m} y_{m}^{-1} x_{m} y_{m}^{-1} x_{m} \ldots\right)=y x_{m}^{-1} y_{m} x_{m}^{-1} y_{m} x_{m}^{-1} y_{m} \ldots
$$

thus defining $k_{x, y}$ everywhere on $\Omega$ in such a way that its action is pointwise approximable by $\Gamma$ almost everywhere. Hence

$$
K=\left\langle k_{x, y}:\{x, y\} \subseteq V^{m}, m \in \mathbb{N}\right\rangle
$$

is a countable group with an ergodic measure-preserving action on $\Omega$ and $K \leq$ $[\Gamma]_{0}$.

We are now in a position to prove our main result.

Theorem 2.8. The von Neumann algebra $L^{\infty}(\Omega) \rtimes \Gamma$ is the hyperfinite factor of type $\mathrm{III}_{1 / n}$. 
Proof. By applying Corollary 2.5 with $G=\Gamma, g \in \Gamma$ any generator of $\Gamma$, and $K$ as in Lemma 2.7 we conclude that $L^{\infty}(\Omega) \rtimes \Gamma$ is a factor of type $\mathrm{III}_{1 / n}$.

To see that the factor is hyperfinite simply note that the action of $\Gamma$ is amenable as a result of [A, Theorem 5.1]. We refer to [C2, Theorem 4.4.1] for the uniqueness of the hyperfinite factor of type $\mathrm{III}_{1 / n}$.

Remark 2.9. In [Sp1], Spielberg constructs $\mathrm{III}_{\lambda}$ factor states on the algebra $\mathcal{O}_{2}$. The reduced $C^{*}$-algebra $C(\Omega) \rtimes_{r} \Gamma$ is a Cuntz-Krieger algebra $\mathcal{O}_{A}$ by [Sp2]. What we have done is construct a type $\mathrm{III}_{1 / n}$ factor state on some of these algebras $\mathcal{O}_{A}$.

Remark 2.10. From $\left[\mathrm{C} 2\right.$, p. 476], we know that if $\Gamma=\mathbb{Q} \rtimes \mathbb{Q}^{*}$ acts naturally on $\mathbb{Q}_{p}$, then the crossed product $L^{\infty}\left(\mathbb{Q}_{p}\right) \rtimes \Gamma$ is the hyperfinite factor of type $\operatorname{III}_{1 / p}$. This may be proved geometrically as above by regarding the the boundary of the homogeneous tree of degree $p+1$ as the one point compactification of $\mathbb{Q}_{p}$ as in $[\mathrm{CKW}]$.

\section{REFERENCES}

[A] S. Adams. Boundary amenability for word hyperbolic groups and an application to smooth dynamics of simple groups. Topology 33 (1994), 765-783.

[CKW] D. I. Cartwright, V. Kaimanovich, and W. Woess. Random walks on the affine group of local fields and homogeneous trees. M.S.R.I. Preprint No. 022-94, 1993. MR 96f:60121

[C1] A. Connes. Une classification des facteurs de type III. Ann. Scient. Ec. Norm. Sup., 6 (1973), pp. 133-252. MR 49:5865

[C2] A. Connes. On the classification of von Neumann algebras and their automorphisms. Symposia Mathematica XX, pp. 435-478, Academic Press 1978. MR 56:9278

[FTN] A. Figà-Talamanca and C. Nebbia, Harmonic Analysis and Representation Theory for Groups Acting on Homogeneous Trees, Cambridge University Press, London 1991. MR 93f: 22004

[HO] T. Hamachi and M. Osikawa, Ergodic Groups Acting of Automorphisms and Krieger's Theorems, Seminar on Mathematical Sciences No. 3, Keio University, Japan, 1981.

[PS] C. Pensavalle and T. Steger, Tensor products and anisotropic principal series representations for free groups, Pac. J. Math. 173 (1996), 181-202.

[S] R. J. Spatzier, An example of an amenable action from geometry, Ergod. Th. \& Dynam. Sys. (1987), 7, 289-293. MR 88j:58100

[Sp1] J. Spielberg, Diagonal states on $\mathcal{O}_{2}$, Pac. J. Math. 144 (1990), 351-382. MR 91k:46065

[Sp2] J. Spielberg, Free product groups, Cuntz-Krieger algebras and covariant maps, Int. J. Math. (1991), 2, 457-476. MR 92j:46120

Department of Mathematics, University of Newcastle, Callaghan, New South Wales 2308, Australia

E-mail address: jacqui@maths.newcastle.edu.au

E-mail address: guyan@maths.newcastle.edu.au 